\title{
Evaluation of Bus Networks in China: From Topology and Transfer Perspectives
}

\author{
Hui Zhang, ${ }^{1}$ Peng Zhao, ${ }^{1}$ Yinhai Wang, ${ }^{2}$ Xiangming Yao, ${ }^{1}$ and Chengxiang Zhuge ${ }^{3}$ \\ ${ }^{1}$ School of Traffic and Transportation, Beijing Jiaotong University, Beijing 100044, China \\ ${ }^{2}$ Civil and Environmental Engineering, University of Washington, Seattle, WA 98195-2700, USA \\ ${ }^{3}$ Department of Geography, University of Cambridge, Cambridge CB2 3EN, UK \\ Correspondence should be addressed to Peng Zhao; bjtu8515@gmail.com
}

Received 11 December 2014; Revised 27 February 2015; Accepted 4 March 2015

Academic Editor: Ricardo López-Ruiz

Copyright (C) 2015 Hui Zhang et al. This is an open access article distributed under the Creative Commons Attribution License, which permits unrestricted use, distribution, and reproduction in any medium, provided the original work is properly cited.

\begin{abstract}
With the development of the public transportation, bus network becomes complicated and hard to evaluate. Transfer time is a vital indicator to evaluate bus network. This paper proposed a method to calculate transfer times using Space P. Four bus networks in China have been studied in this paper. Some static properties based on graph theory and complex theory are used to evaluate bus topological structure. Moreover, a bus network evolution model to reduce transfer time is proposed by adding lines. The adding method includes four types among nodes with random choice, large transfer time, degree, and small degree. The results show that adding lines with nodes of small degree is most effective comparing with the other three types.
\end{abstract}

\section{Introduction}

Bus network plays more and more significant role in alleviating the increasingly heavy traffic congestion in metropolis due to urban sprawl and population explosion. During the last two decades, there are many researches on transportation systems using complex networks theory such as subway networks [1-3], aviation networks $[4,5]$, marine networks [6], street networks [7-9], railway networks [10,11], highway networks [12], and commuting networks [13]. Many studies focus on some static properties of transit networks such as degree distribution, the average shortest path distance, and cluster [14-17].

Complex network theory is a useful tool to analyze network topological structures. It has been successfully applied in many fields including power grid networks $[18,19]$, scientific collaboration networks [20-22], WWW $[23,24]$, and protein networks [25-27] in attempt to understand the relationship between topological properties and performances. The topological structure and dynamics are two aspects of a complex system and they interact with each other [28, 29]. An eminent topological structure can always bring highperformance dynamics. Although there are many researches on the analysis of bus topological structure, seldom research focuses on the transfer issues. In bus network, transfer is such a very important factor that it cannot be negligible. Because of time-consuming and inconvenience, transfer has become a barrier to hinder passengers from using bus network. How to reduce transfer times has become a priority problem to resolve for transit network designer and planner.

As a complex network, bus network has its unique traits. Lines play an important role in constituting a bus network; it is necessary to understand the effect of each line. To evaluate the topological structures of bus networks, this paper uses several indicators based on graph theory and complex theory and proposes two indicators concerning transfers. An effective method to calculate the average transfer time considering traffic demand has been proposed using Space P. Moreover, the role of each line playing in transfer times of the whole network has been studied. In the end, four types to reduce total transfer time have been given by adding lines.

This paper focuses on the transfer issues of bus network and aims to find an efficient way to reduce transfer times. The rest of paper is organized as follows. Section 2 describes bus network representation. Statistic properties are given in Section 3. Section 4 gives the two transfer-related indicators. 


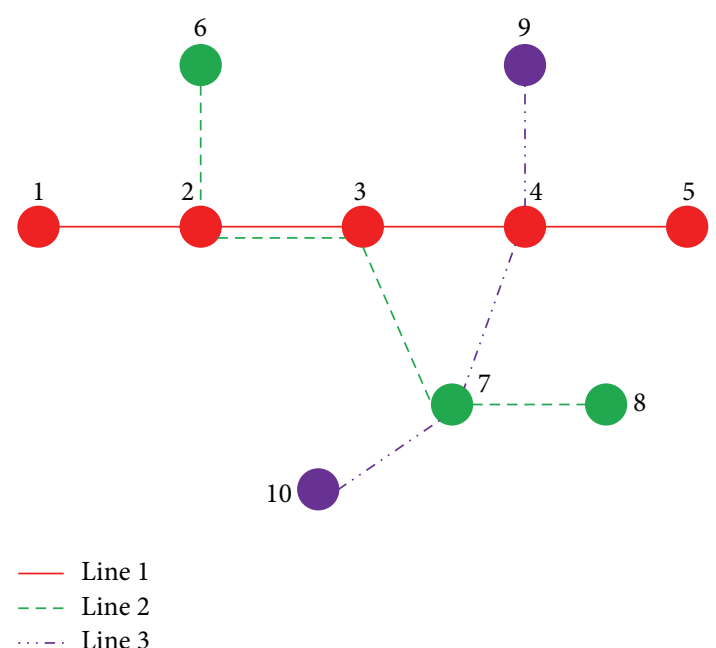

(a)

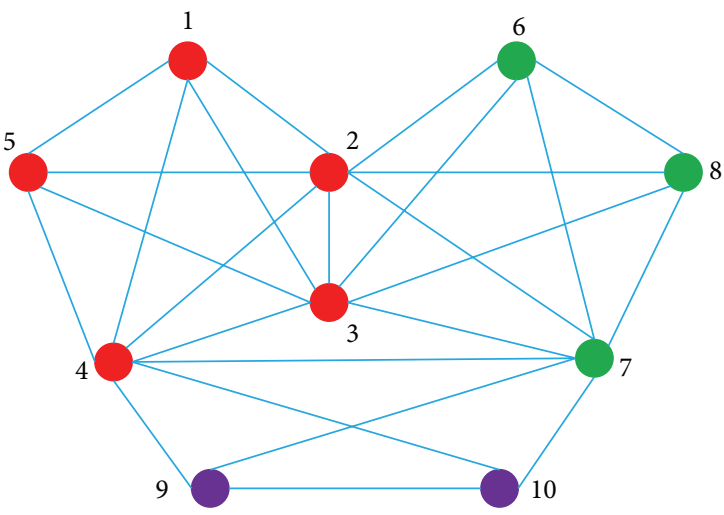

(b)

FIGURE 1: Simple bus network described by Space P.

The evolution of bus network transfer is introduced in Section 5.

\section{The Approaches of Describing Bus Network}

In this paper, we use three forms of matrices to represent the bus network: line-station matrix, weighted adjacent matrix, and adjacent matrix under Space P. Line-station matrix is a basic form to express bus network, where each row stands for a line and each column stands for a station. Take Figure 1(a) for example; there are three rows in the matrix: (1) 1-2-34-5, (2) 6-2-3-7-8, and (3) 9-4-7-10. The weighted adjacent matrix is widely used to present networks in graph theory. Here, it is used to calculate some indicators of bus network. We represent networks as $G=(V, E)$, where $V$ is the set of nodes and $E$ is the set of edges. $G$ is described by the $N \times N$ adjacent matrix $\left\{a_{i j}\right\} ; a_{i j}$ is the weight of edge between nodes $i$ and $j$. If there is no edge between nodes $i$ and $j, a_{i j}=0$. When the weight equals 1 , it is the common adjacent matrix. In this paper, there are two weights to be used, which are the section length and the number of overlapped section. Another useful matrix to display bus network is adjacent matrix under Space $\mathrm{P}$. In this matrix, nodes which belong to a line connect with each other (see Figure 1(b)).

\section{The Basic Properties}

Baoding, Jinan, Shijiazhuang, and Suzhou are four medium cities in China. As the economy developed rapidly in these years, the demand of public transportation is high. We collected the four cities bus data from website in 2014. The data contain line name, station name, the distance between two adjacent stations, and the number of overlapped edges between two adjacent stations. Totally, there are 52 lines and 634 stations in Baoding, 100 lines and 883 stations in Jinan, 139 lines and 1299 stations in Shijiazhuang, and 109 lines and 1402 stations in Suzhou. Figure 2 shows the topological structures of the four cities. The size of nodes stands for the degree of the nodes; the large size means large degree. The colors stand for community structures. The specific values of indicators are shown in Table 1.

Gamma Index. Gamma index is used to measure network connectivity for planar graphs in graph theory [30], which is denoted as $\gamma=e /(3(v-2))$, where $\gamma$ is gamma index, $v$ is the number of nodes, and $e$ is the number of edges. The higher the gamma index is the densely connected the network is. Up to this point, the range of gamma index of the four cites is $[0.4,0.5]$. Obviously, the connectivity of Shijiazhuang is better than the other three.

Average Line Length. Average line length is an indicator to reflect bus line span, which is calculated as $\bar{L}=\left(\sum_{i=1}^{N L} L_{i}\right) / N L$, where $\bar{L}$ is the average line length, $N L$ is the number of lines, and $L_{i}$ is the $i$ th line length. Average line length is related with transfer time and bus running time.

Line Overlapping Degree. Line overlapping degree is the ratio between the total overlapped link length and total network length, which is expressed as

$$
\theta=\frac{\sum_{m}(u-1) l_{m}^{u}}{\sum_{i=1}^{N L} L_{i}},
$$

where $\theta$ is the line overlapping degree, $u$ is the multiplicity of edge $m$, and $l_{m}^{u}$ is the length of edge $m$ with multiplicity $u$. The overlapping degree reflects the situation of overlapped edges in the networks; large value of line overlapping degree means more overlapped edges.

Degree. Degree is an important index in complex networks. The node $i$ degree $k_{i}$ is defined as the number of nodes that connect with it. The degree distribution probability of many networks follows $p(k)=k^{-\tau}$ with $2<\tau \leq 3$ [31]. The average degree $\bar{k}$ reflects the basic properties of complex network. 
TABLE 1: Statistical properties values of gamma index, average line length, line overlapping degree, average degree, efficiency, degree correlation, and modularity for Baoding, Jinan, Shijiazhuang, and Suzhou.

\begin{tabular}{lccccccc}
\hline Indicators & $\gamma$ & $\bar{L}$ & $\theta$ & $\bar{k}$ & $E$ & \multicolumn{1}{c}{$\mathrm{Q}$} \\
\hline Baoding & 0.4655 & 15.5 & 0.437 & 2.784 & 0.1011 & 0.1166 & 0.838 \\
Jinan & 0.4431 & 13.4 & 0.476 & 2.652 & 0.0724 & 0.2661 & 0.860 \\
Shijiazhuang & 0.4868 & 15.9 & 0.435 & 2.916 & 0.0901 & 0.1950 \\
Suzhou & 0.4860 & 22.2 & 0.447 & 2.912 & 0.0742 & 0.1685 & 0.854 \\
\hline
\end{tabular}
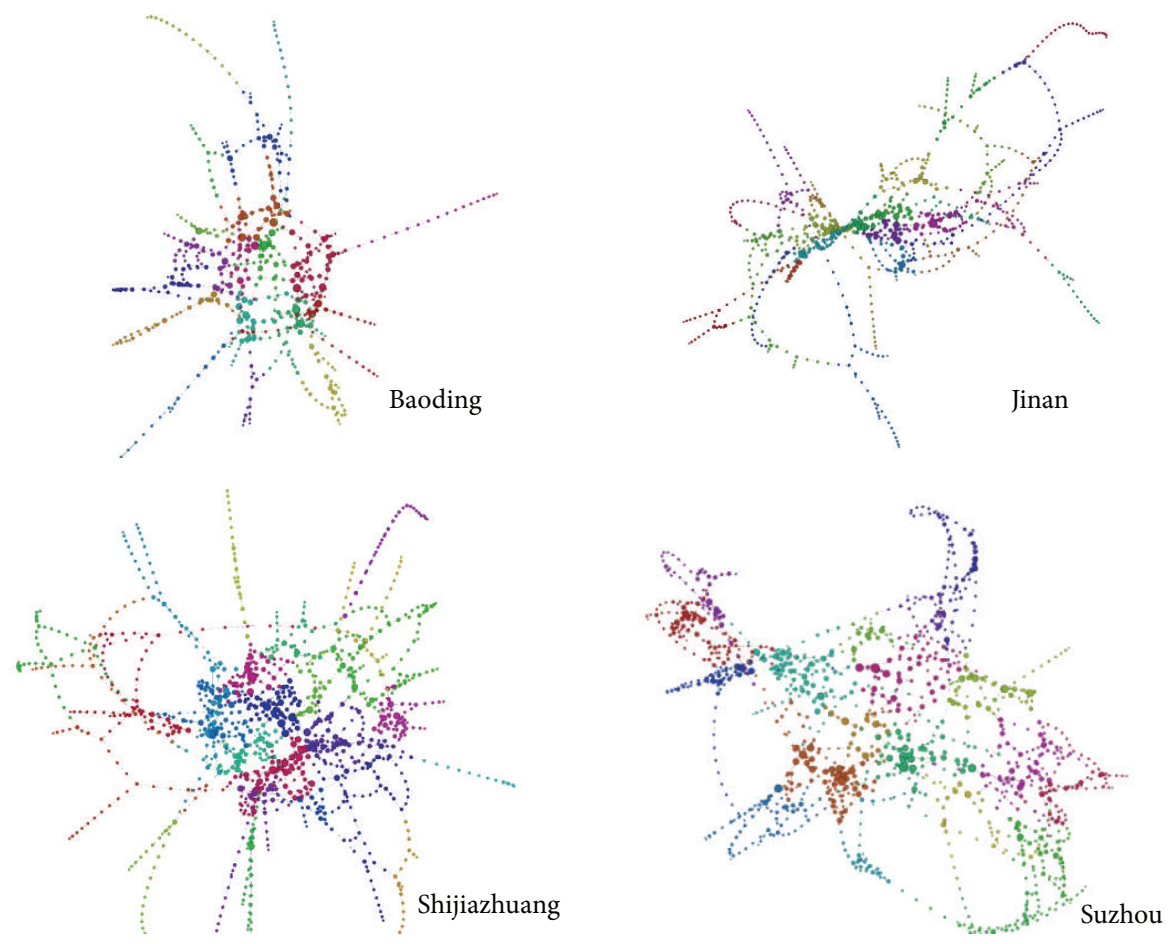

Figure 2: The topological structures of transit networks of Baoding, Jinan, Shijiazhuang, and Suzhou. The colors represent communities of transit network; the size of nodes represents the degree.

The average degree of Baoding, Jinan, Shijiazhuang, and Suzhou is $2.784,2.652,2.916$, and 2.912, which is slightly larger than subway networks [32].

Efficiency. Efficiency is the property to measure the capacity of network. It is denoted as $E=2 * \sum_{i>j}\left(1 / s_{i j}\right) /(N(N-1))$, where $E$ is efficiency and $s_{i j}$ is the shortest path between node $i$ and node $j$. Large value indicates good performance of network.

Degree Correlation. Degree correlation reflects the connecting mode between nodes with different degree. In networks, nodes with high degree which have the tendency to connect nodes with high degree are called assortativity. By contrast, nodes with high degree tending to connect nodes with low degree are called disassortativity. Degree correlation is an important key feature and plays a key role in complex networks [33, 34]. It can be expressed with the formula [35]

$$
r=\frac{M^{-1} \sum_{i} j_{i} k_{i}-\left[M^{-1} \sum_{i}(1 / 2)\left(j_{i}+k_{i}\right)\right]^{2}}{M^{-1} \sum_{i}(1 / 2)\left(j_{i}^{2}+k_{i}^{2}\right)-\left[M^{-1} \sum_{i}(1 / 2)\left(j_{i}+k_{i}\right)\right]^{2}},
$$

where $r$ is the correlation coefficient and $j_{i}$ and $k_{i}$ are the degree of the nodes of $i$ th edge, with $i=1, \ldots, M . r>0$ means assortative network and $r<0$ means disassortative network.

Community Structure. Community structure is also an important characteristic in transportation networks and plays a significant role in traffic dynamics [28]. It is the structure where there are many links in the community but fewer between communities [36, 37]. We can use modularity to calculate the level of community. It is defined as [38] $Q=$ $\sum_{i} e_{i j}-\sum_{i j k} e_{i j} e_{k i}=T r e-\left\|e^{2}\right\|$, where $\|x\|$ means the sum of all elements of $x$. We can get that $0 \leq Q \leq 1$; the larger the modularity is the stronger the community structure is. We can see, from Figure 2, there are 19, 22, 23, and 19 communities in Baoding, Jinan, Shijiazhuang, and Suzhou.

From Table 1, we can see that the city of Shijiazhuang has the biggest value of gamma indicator, but the efficiency value is smaller than the city of Baoding. The results show that good structure is more important than the number of edges. Moreover, the results also show that the bus networks 
are assortative correlated networks with degree correlation of $0.1166,0.2661,0.1950$, and 0.1685 for Baoding, Jinan, Shijiazhuang, and Suzhou.

\section{Methods to Calculate Transfer-Related Indicators}

Besides the aforementioned indicators, this paper focuses on the transfer-related indicators such as the average transfer time of the whole transit network and transfer disutility.

4.1. The Average Transfer Time. The average transfer time is a significant predictor to evaluate transit network. Intuitively, the higher the average transfer time is the worse the transit performance is. It is hard to calculate the transfer time among the network using the traditional line-station matrix. Here, the adjacent matrix under Space $\mathrm{P}$ is used to calculate the average transfer times considering traffic demand. It is calculated with the formula

$$
\mathrm{ATT}=\sum_{i=1}^{N} \sum_{j=1}^{N} \frac{d_{i j}\left(s_{i j}^{P}-1\right)}{(N(N-1))}
$$

where ATT is the average transfer time, $d_{i j}$ is the traffic demand between node $i$ and node $j, s_{i j}^{P}$ is the shortest path distance between node $i$ and node $j$ under Space P. In this paper, we focus on the structure of bus network, without considering traffic demand; let all $d_{i j}=1$.

4.2. Transfer Disutility. Transfer disutility is correlated with transfer time and degree. It can be expressed as

$$
\xi=\frac{\left(\sum_{i=1}^{N} \sum_{j=1}^{N}\left(\left(1+t t_{i j}\right) /\left(k_{i}^{2}+k_{j}^{2}\right)\right)\right)}{(N(N-1))}
$$

where $\xi$ is transfer disutility and $t t_{i j}$ is transfer time between node $i$ and node $j$. Complex network researches show that most transit networks are assortative correlation networks, which means nodes with large degree tend to connect nodes with large degree directly. Transfer disutility can reflect the transfer mechanism in transit network based on complex network theory. Small value of transfer disutility means good performance of structure.

Based on the two formulas, the average transfer times of Baoding, Jinan, Shijiazhuang, and Suzhou are 1.0712, 1.3138, 1.4640 , and 1.2416 which indicates that the structure of Baoding outperforms the other three. The specific transfer time proportions are given in Table 2; the proportion of transfer time is the number of OD pairs of the transfer time divided by total number OD pairs. By contrast, the transfer disutility is $0.101,0.131,0.125$, and 0.110 which also validate that the performance of Baoding is the best among the four bus structures.
TABLE 2: The proportion of transfer times of the four cities.

\begin{tabular}{lcccc}
\hline Transfer time & 0 & 1 & 2 & 3 and more \\
\hline Baoding & 0.0946 & 0.7396 & 0.1658 & 0 \\
Jinan & 0.0698 & 0.5588 & 0.3567 & 0.0147 \\
Shijiazhuang & 0.0407 & 0.4698 & 0.4724 & 0.0171 \\
Suzhou & 0.0626 & 0.6337 & 0.3016 & 0.0030 \\
\hline
\end{tabular}

\section{Evolution on Bus Networks to Reduce Transfer}

In order to further study the particularity of transfer of bus network, this paper puts emphasis on the transfer between different lines and evolution on transfer by adding lines. Bus network is comprised of lines as basic components, not edges. Therefore, transfer times between lines are so important when evaluating bus network. The transfer times between lines can be calculated as $l t t_{i j}=\sum_{m \in l_{i}} \sum_{n \in l_{j}} t t_{m n}$, where $l t t_{i j}$ is transfer times between line $i$ and line $j$ and $m$ and $n$ are nodes that belong to line $l_{i}$ and $l_{j}$. Figure 3 shows the transfer time between lines in the four cities. As we can see, there are some lines with high transfers to other lines. For example, the most transfer time lines are number 13 , number 52, number 113, and number 50 for Baoding, Jinan, Shijiazhuang, and Suzhou with $66257,135397,196664$, and 230366 times, respectively.

In a bus network, some lines are indispensable to reduce the total transfer times. In order to better assess the importance of a line of bus network, each line was deleted separately from the network. Intuitively, the total transfer time of bus network will increase or remain the same after deleting a line. Here, we calculate the ratio between the original total transfer times and modified total transfer times using the formula

$$
\rho_{k}=\frac{\sum_{i \in N} \sum_{j \in N} t t_{i j}}{\sum_{i \in N^{\prime}} \sum_{j \in N^{\prime}} t t_{i j}^{k}}
$$

where $\rho_{k}$ is the proportion after deleting $k$ th line, $N$ is the set of total number of nodes, $N^{\prime}$ is the set of total nodes after deleting a line, and $t t_{i j}^{k}$ is the transfer times after deleting $k$ th line. Note that there may be some nodes that will not connect with the network after deleting lines. Here, the transfer times between separated nodes and other nodes are preset to 5 after deleting a line.

As we can see from Figure 4, the proportions are less than 1, which means the total transfer times increase after deleting a line. Moreover, there are some lines playing an important role in transfer times of bus network. For example, the most important lines of bus networks are number 13, number 52, number 113, and number 50 of Baoding, Jinan, Shijiazhuang, and Suzhou with the proportions of 0.9324 , $0.9593,0.9730$, and 0.9722 . The result also validates the transfer times between lines.

To reduce the transfer times and increase the competitiveness, it is necessary to understand the evolution of bus network. In this part, we seek to reduce transfer times of bus network by adding edges. The aim is to find an effective way to reduce the transfer times. The specific processes are as follows: 20 lines will be added gradually to the network 


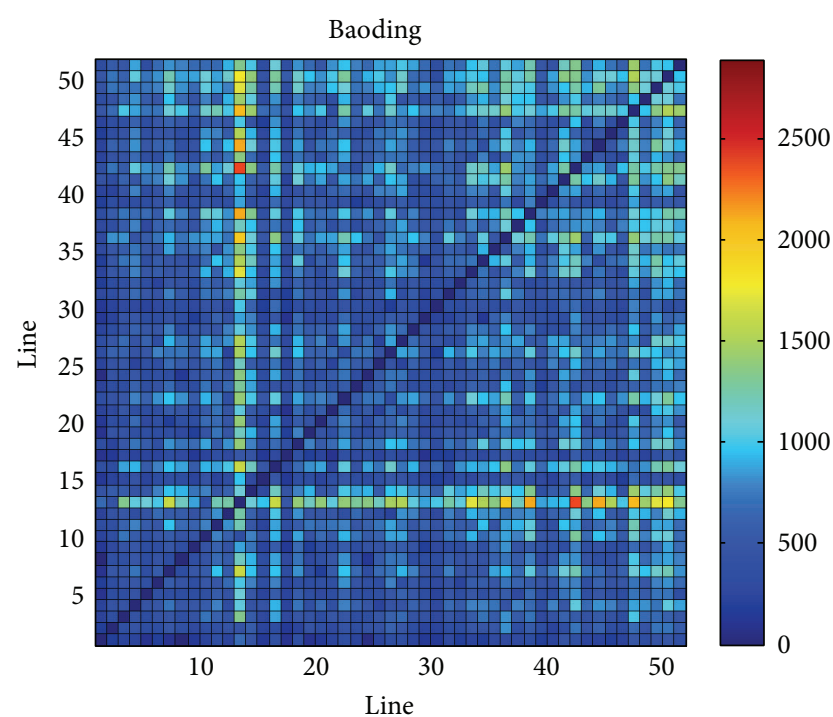

(a)

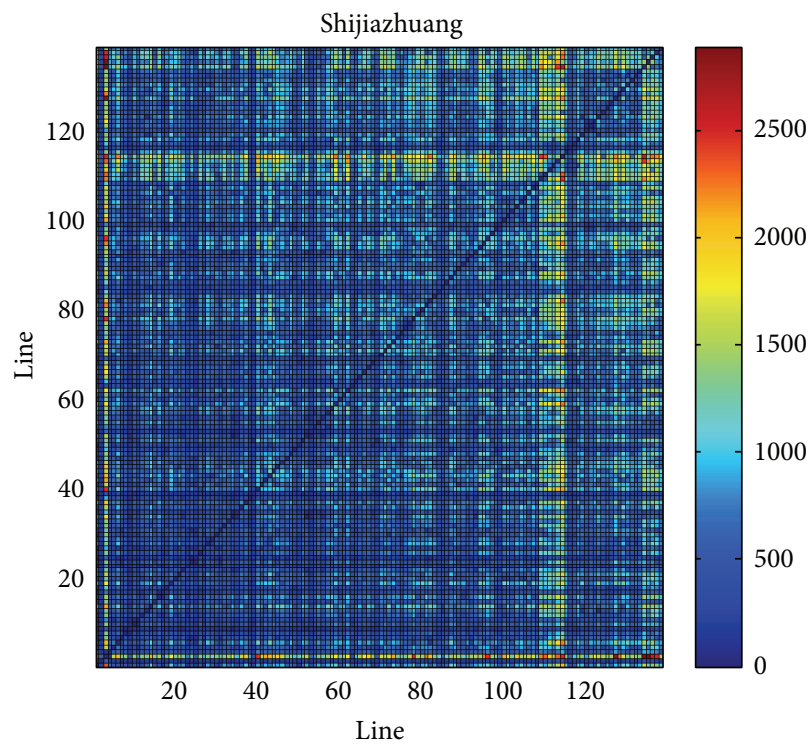

(c)

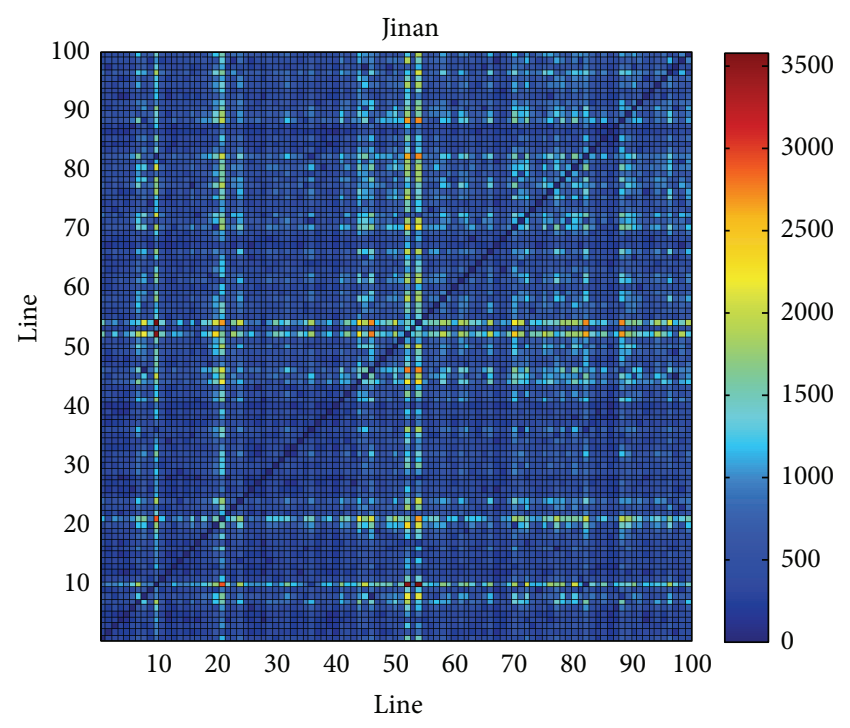

(b)

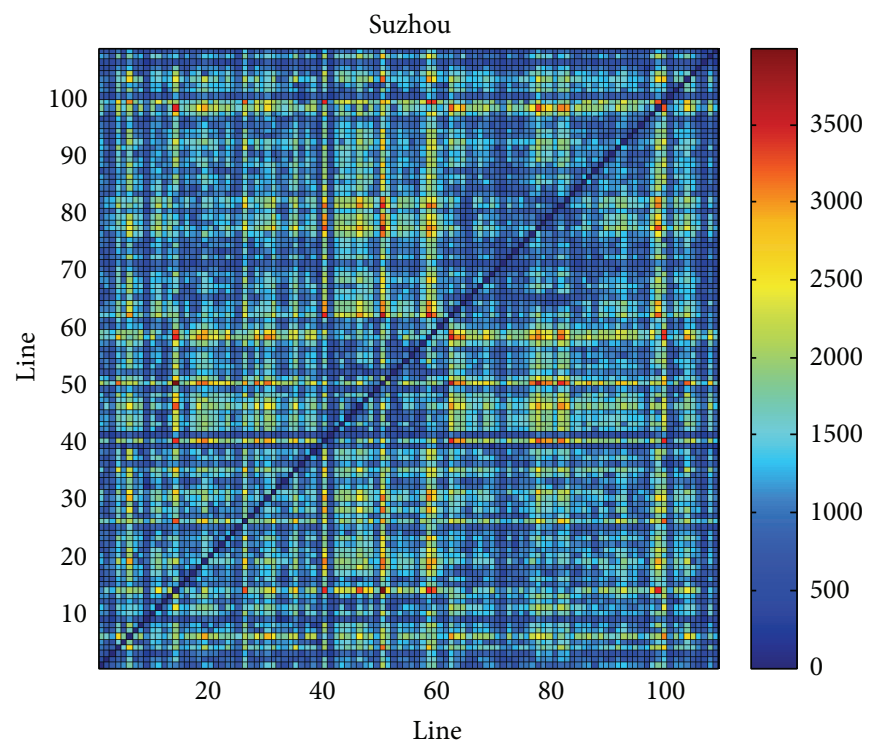

(d)

FIgURE 3: The total transfer times between any two lines.

with four-type principles. Each line contains 20 nodes. The principles to choose nodes are based on (1) random choice, (2) the nodes with largest transfer times, (3) the nodes with largest degree, and (4) the nodes with smallest degree. Take principle (2), for example; at each step, the 20 nodes with largest transfer times are picked out and connect with each other as a line. Then recalculate the transfer times and new 20 nodes are picked out until 20 lines have been added. The ratio between original total transfer times and modified total transfer time is given with the formula

$$
\Omega_{m}=\frac{\sum_{i \in N} \sum_{j \in N} t t_{i j}^{m}}{\sum_{i \in N} \sum_{j \in N} t t_{i j}},
$$

where $\Omega_{m}$ and $t t_{i j}^{m}$ are the transfer ratio and transfer times between node $i$ and node $j$ after adding $m$ th lines, respectively.

As can be seen from Figure 5, the ratios decrease after adding lines. Take Baoding, for example; the transfer ratios with adding lines among small degree are 0.9936, 0.9661, and 0.9488 at step 1 , step 10, and step 20. It is obvious that adding lines with nodes of smallest degree is the most effective way to reduce total network transfer times. For example, the ratios of random choice, large transfer time, large degree, and small degree in Baoding are 0.9654, 0.9759, 0.9516, and 0.9488, respectively. It is noted that the ratio of principle of adding lines with large degree decreases slowly at first several steps, and the ratio of principle with small degree decreases fast at first several steps. However, the ratios under two principles 


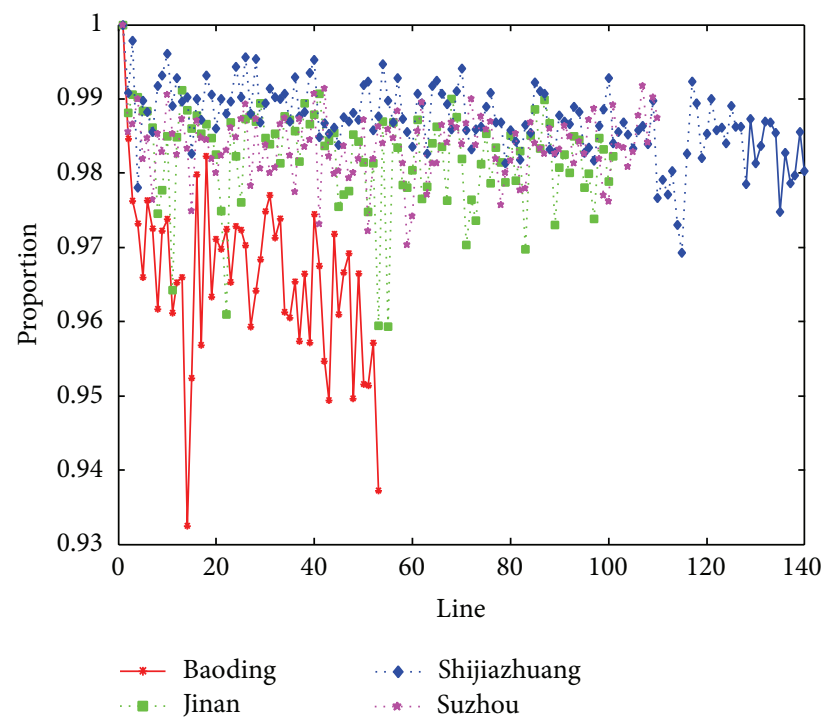

FIGURE 4: The total transfer proportion after deleting a line.

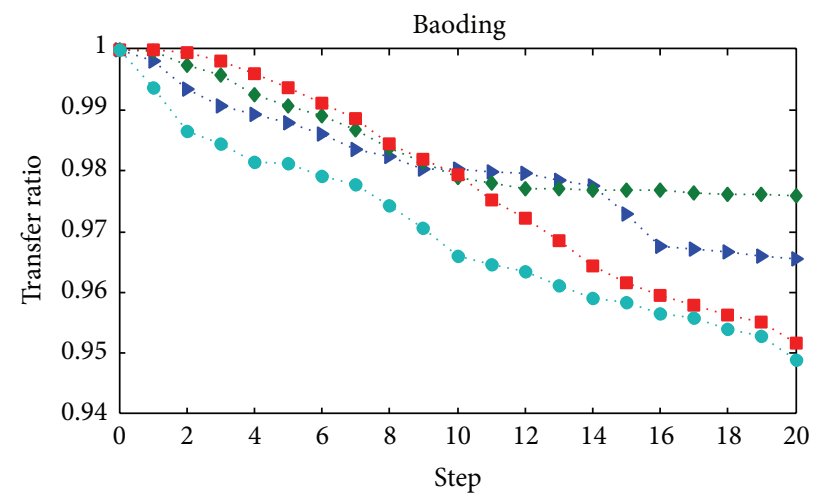

(a)

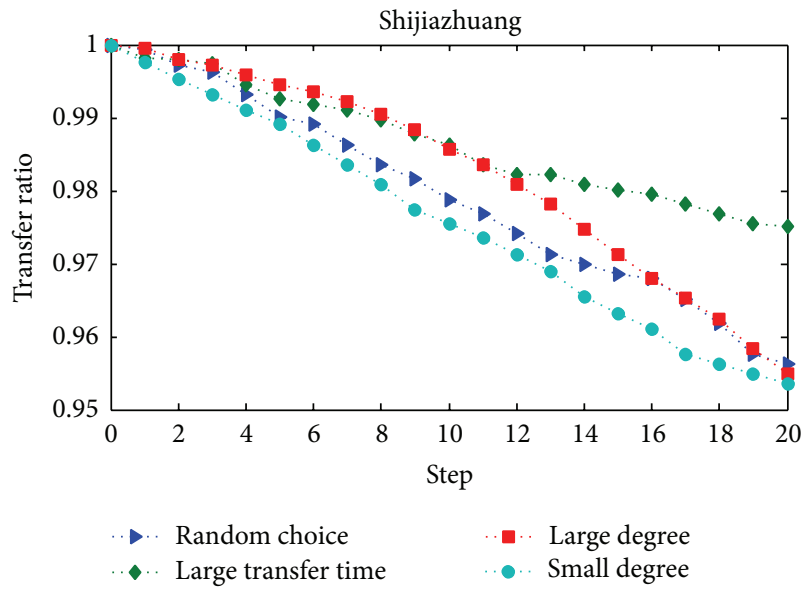

(c)

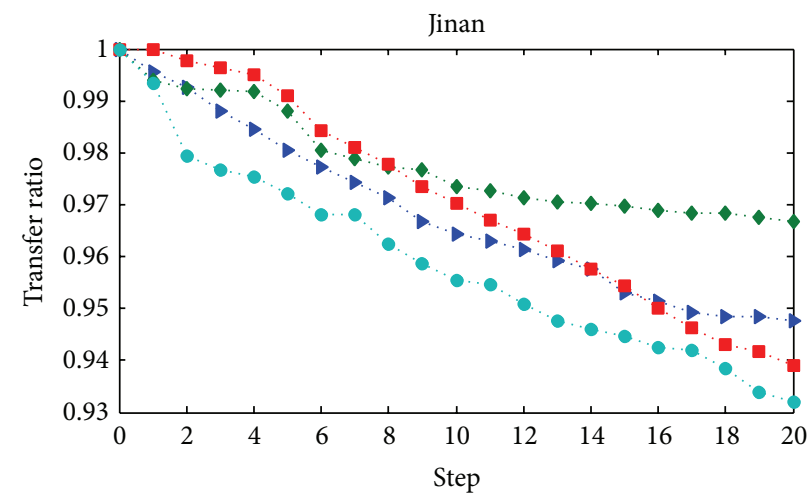

(b)

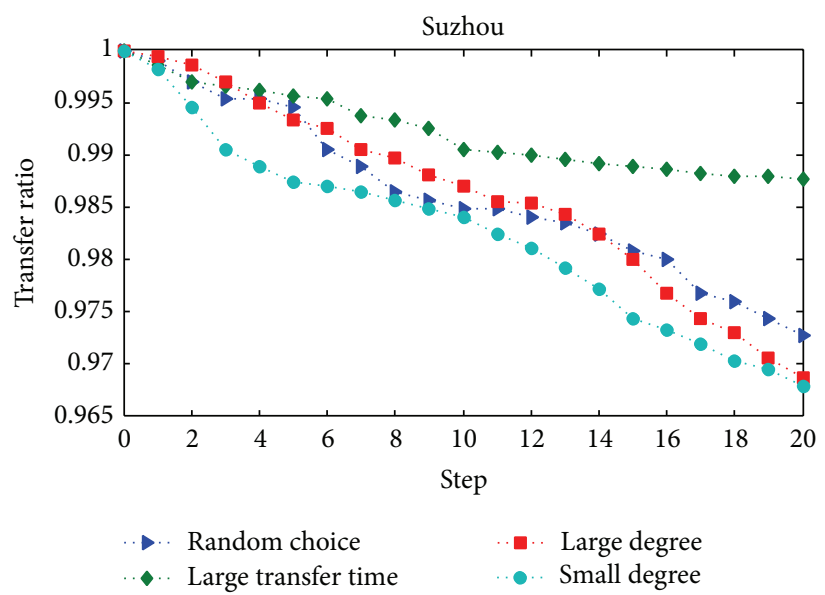

(d)

FIGURE 5: The transfer ratio with four adding edges modes: random choice, large transfer time, large degree, and small degree. 
get close at the last steps. The reasons are that nodes with large degree have always been connected and adding links cannot decrease transfer times effectively. By contrast, nodes with small degree connected sparsely; adding lines can effectively reduce total network transfer times. At the last steps, it is hard to reduce transfer times obviously because degrees of nodes picked out under two principles have no distinct differences. From the figure, it is surprising to find that the principle of adding lines with large transfer time cannot reduce transfer time effectively, which indicates connecting those nodes with large transfer times cannot give large contribution to the total network structure.

\section{Conclusion}

This paper studied the evaluation of bus networks from topology and transfer perspectives. The average transfer time has an important impact on transit performance. In this paper, the method to calculate transfer time between any two nodes with different traffic demand using Space $\mathrm{P}$ has been proposed. Statistical properties show that bus networks are assortative network with multiple communities.

Lines play a significant role in comprising bus network. This paper studied the role of each line of networks by deleting lines. The results show there are several lines in the network which has important influence on transfer times. In order to reduce transfer times, four types of adding lines are applied in the four networks. Four types mean four different nodes choosing methods: random choice, large transfer time, large degree, and small degree. The results show that adding lines among nodes with small degree has the best effect comparing to the other three. The future work should consider the practical traffic demand between any two nodes and other influential factors.

\section{Conflict of Interests}

The authors declare that there is no conflict of interests regarding the publication of this paper.

\section{Acknowledgment}

This paper is supported by National Natural Science Foundation of China (51278030 and 51478036).

\section{References}

[1] C. Roth, S. M. Kang, M. Batty, and M. Barthelemy, "A long-time limit for world subway networks," Journal of the Royal Society Interface, vol. 9, no. 75, pp. 2540-2550, 2012.

[2] B. Leng, X. Zhao, and Z. Xiong, "Evaluating the evolution of subway networks: evidence from Beijing subway network," $E P L$, vol. 105, Article ID 58004, 6 pages, 2014.

[3] B. Ek, C. VerSchneider, and D. A. Narayan, "Efficiency of star-like graphs and the Atlanta subway network," Physica A. Statistical Mechanics and its Applications, vol. 392, no. 21, pp. 5481-5489, 2013.
[4] J. Lin, "Network analysis of China's aviation system, statistical and spatial structure," Journal of Transport Geography, vol. 22, pp. 109-117, 2012.

[5] T. Jia, K. Qin, and J. Shan, "An exploratory analysis on the evolution of the US airport network," Physica A: Statistical Mechanics and Its Applications, vol. 413, pp. 266-279, 2014.

[6] Y. H. Hu and D. L. Zhu, "Empirical analysis of the worldwide maritime transportation network," Physica A: Statistical Mechanics and Its Applications, vol. 388, no. 10, pp. 2061-2071, 2009.

[7] M. Barthélemy and A. Flammini, "Modeling Urban street patterns," Physical Review Letters, vol. 100, no. 13, Article ID 138702, 4 pages, 2008.

[8] Y.-S. Qian, M. Wang, H.-X. Kang, J.-W. Zeng, and Y.-F. Liu, "Study on the road network connectivity reliability of valley city based on complex network," Mathematical Problems in Engineering, vol. 2012, Article ID 430785, 14 pages, 2012.

[9] Y. Y. Duan and F. Lu, "Robustness of city road networks at different granularities," Physica A: Statistical Mechanics and its Applications, vol. 411, pp. 21-34, 2014.

[10] M. Kurant and P. Thiran, "Extraction and analysis of traffic and topologies of transportation networks," Physical Review E: Statistical, Nonlinear, and Soft Matter Physics, vol. 74, no. 3, Article ID 036114, 2006.

[11] W. Li and X. Cai, "Empirical analysis of a scale-free railway network in China," Physica A: Statistical Mechanics and its Applications, vol. 382, no. 2, pp. 693-703, 2007.

[12] W.-S. Jung, F. Wang, and H. E. Stanley, "Gravity model in the Korean highway," Europhysics Letters, vol. 81, no. 4, Article ID 48005, 6 pages, 2008.

[13] V. De Leo, G. Santoboni, F. Cerina, M. Mureddu, L. Secchi, and A. Chessa, "Community core detection in transportation networks," Physical Review E, vol. 88, no. 4, Article ID 042810, 2013.

[14] C. von Ferber, T. Holovatch, Y. Holovatch, and V. Palchykov, "Public transport networks: empirical analysis and modeling," European Physical Journal B, vol. 68, no. 2, pp. 261-275, 2009.

[15] X. Zheng, J. P. Chen, J. L. Shao, and L. D. Bie, "Analysis on topological properties of Beijing urban public transit based on complex network theory," Acta Physica Sinica, vol. 61, no. 19, Article ID 190510, 2012.

[16] X. Xu, J. Hu, F. Liu, and L. Liu, "Scaling and correlations in three bus-transport networks of China," Physica A: Statistical Mechanics and its Applications, vol. 374, no. 1, pp. 441-448, 2007.

[17] Y. T. Mohmand and A. Wang, "Complex network analysis of Pakistan railways," Discrete Dynamics in Nature and Society, vol. 2014, Article ID 126261, 5 pages, 2014.

[18] E. Cotilla-Sanchez, P. D. H. Hines, C. Barrows, and S. Blumsack, "Comparing the topological and electrical structure of the North American electric power infrastructure," IEEE Systems Journal, vol. 6, no. 4, pp. 616-626, 2012.

[19] Y. D. Zhang, Z. J. Bao, Y. J. Cao, G. Li, and G. Chen, "Long-term effect of different topology evolutions on blackouts in power grid," International Journal of Electrical Power \& Energy Systems, vol. 62, pp. 718-726, 2014.

[20] M. E. Newman, "The structure of scientific collaboration networks," Proceedings of the National Academy of Sciences of the United States of America, vol. 98, no. 2, pp. 404-409, 2001.

[21] R. K. Pan and J. Saramäki, "The strength of strong ties in scientific collaboration networks," Europhysics Letters, vol. 97, no. 1, Article ID 18007, 2012. 
[22] X. F. Liu, X.-K. Xu, M. Small, and C. K. Tse, "Attack resilience of the evolving scientific collaboration network," PLOS ONE, vol. 6, no. 10, Article ID e26271, 7 pages, 2011.

[23] A.-L. Barabási and R. Albert, "Emergence of scaling in random networks," Science, vol. 286, no. 5439, pp. 509-512, 1999.

[24] N. Perra, V. Zlatić, A. Chessa, C. Conti, D. Donato, and G. Caldarelli, "PageRank equation and localization in the WWW", Europhysics Letters, vol. 88, no. 4, Article ID 48002, 2009.

[25] S. Maslov and K. Sneppen, "Specificity and stability in topology of protein networks," Science, vol. 296, no. 5569, pp. 910-913, 2002.

[26] C. M. Schneider, L. de Arcangelis, and H. J. Herrmann, "Modeling the topology of protein interaction networks," Physical Review E-Statistical, Nonlinear, and Soft Matter Physics, vol. 84, no. 1, Article ID 016112, 2011.

[27] K. Trivodaliev, A. Bogojeska, and L. Kocarev, "Exploring function prediction in protein interaction networks via clustering methods," PLoS ONE, vol. 9, no. 6, Article ID e99755, 2014.

[28] H.-J. Sun, H. Zhang, and J.-J. Wu, "Correlated scale-free network with community: modeling and transportation dynamics," Nonlinear Dynamics, vol. 69, no. 4, pp. 2097-2104, 2012.

[29] S. Havlin, D. Y. Kenett, A. Bashan, J. Gao, and H. E. Stanley, "Vulnerability of network of networks," The European Physical Journal Special Topics, vol. 223, no. 11, pp. 2087-2106, 2014.

[30] D. Gattuso and E. Miriello, "Compared analysis of metro networks supported by graph theory," Networks and Spatial Economics, vol. 5, no. 4, pp. 395-414, 2005.

[31] L. A. N. Amaral, A. Scala, M. Barthélémy, and H. E. Stanley, "Classes of small-world networks," Proceedings of the National Academy of Sciences of the United States of America, vol. 97, no. 21, pp. 11149-11152, 2000.

[32] J. Zhang, M. Zhao, H. Liu, and X. Xu, "Networked characteristics of the urban rail transit networks," Physica A: Statistical Mechanics and its Applications, vol. 392, no. 6, pp. 1538-1546, 2013.

[33] H.-B. Hu and X.-F. Wang, "Disassortative mixing in online social networks," Europhysics Letters, vol. 86, no. 1, Article ID 18003, 6 pages, 2009.

[34] S.-M. Cai, Y.-B. Zhou, T. Zhou, and P.-L. Zhou, "Hierarchical organization and disassortative mixing of correlation-based weighted financial networks," International Journal of Modern Physics C, vol. 21, no. 3, pp. 433-441, 2010.

[35] M. E. J. Newman, “Assortative mixing in networks," Physical Review Letters, vol. 89, Article ID 208701, 4 pages, 2002.

[36] C. Tong, J. W. Niu, Z. Y. Xie, and F. Peng, "Sampling from social network to maintain community structure," International Journal of Communication Systems, vol. 27, no. 9, pp. 1363-1377, 2014.

[37] M.-Y. Zhou, Z. Zhuo, S.-M. Cai, and Z. Q. Fu, "Community structure revealed by phase locking," Chaos, vol. 24, no. 3, Article ID 033128, 7 pages, 2014.

[38] M. E. J. Newman and M. Girvan, "Finding and evaluating community structure in networks," Physical Review E: Statistical, Nonlinear, and Soft Matter Physics, vol. 69, no. 2, Article ID 026113, 2004. 


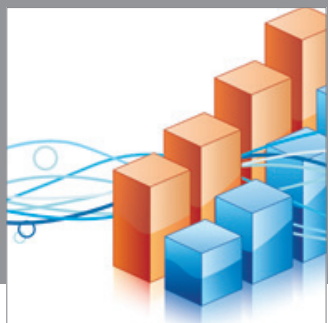

Advances in

Operations Research

mansans

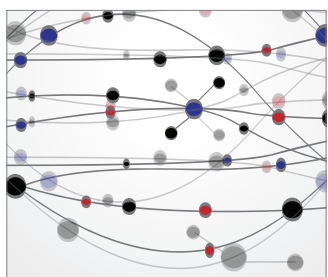

The Scientific World Journal
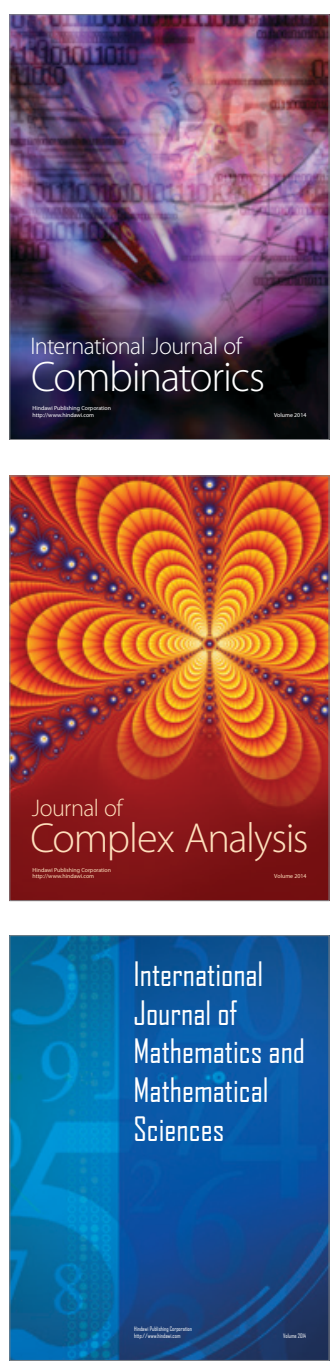
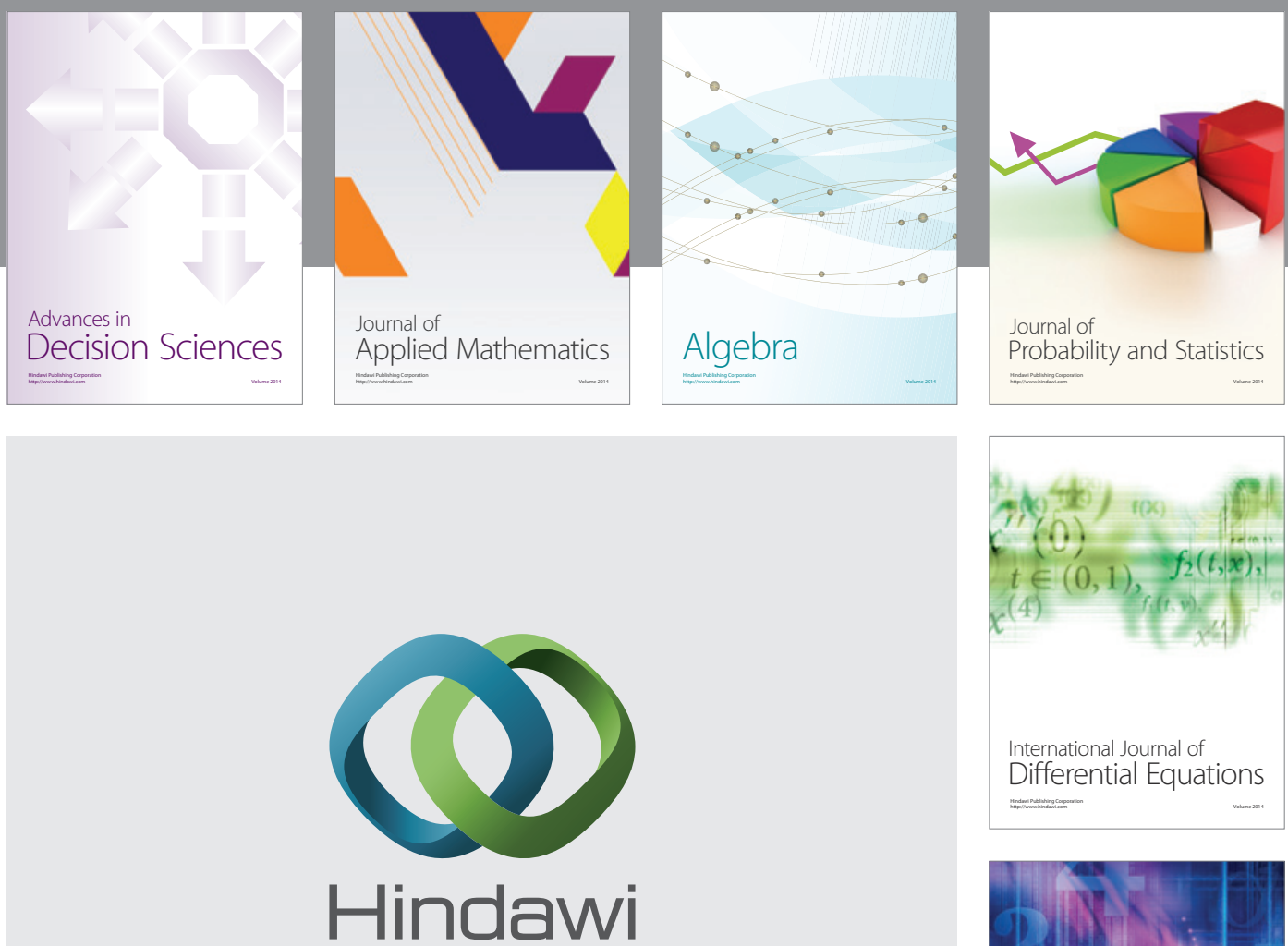

Submit your manuscripts at http://www.hindawi.com
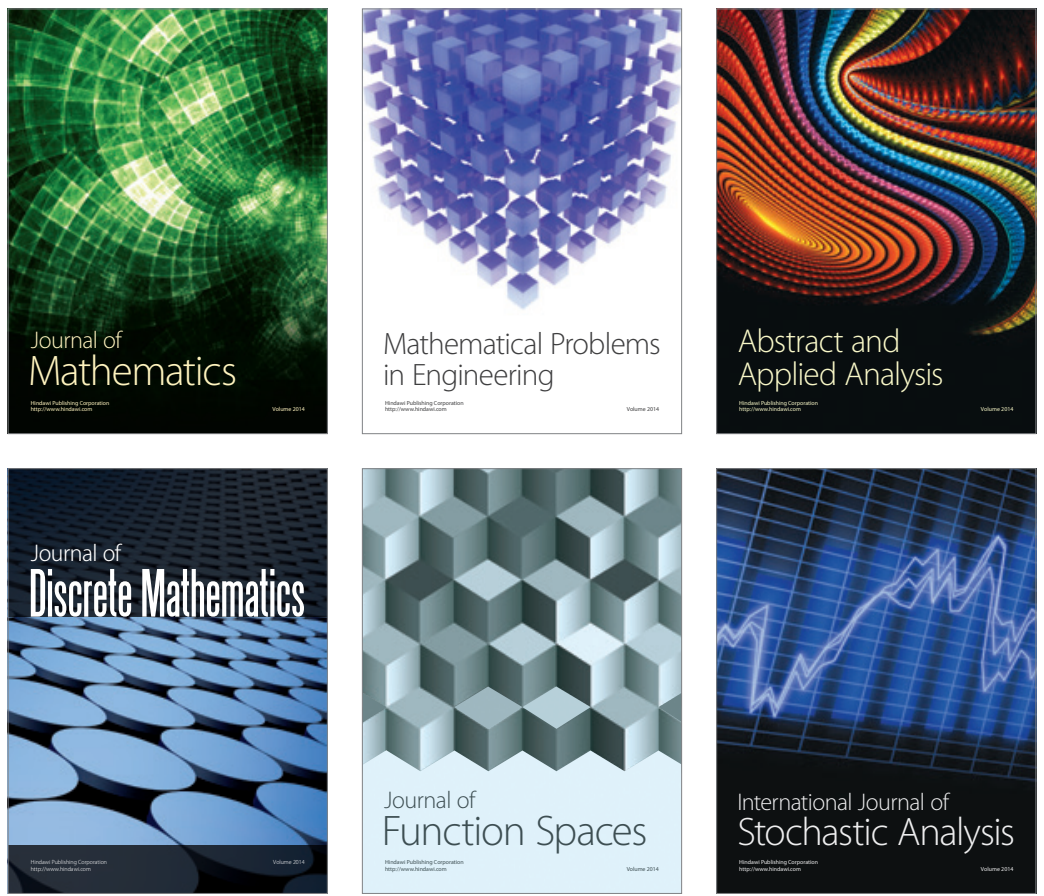

Journal of

Function Spaces

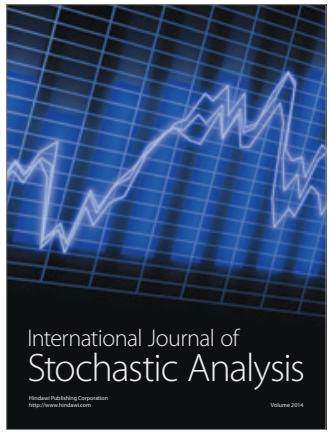

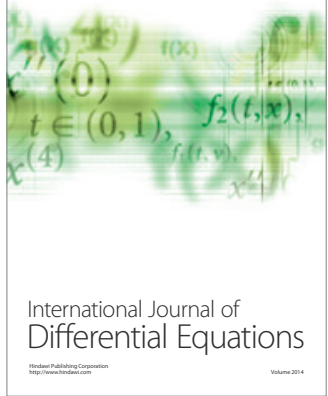
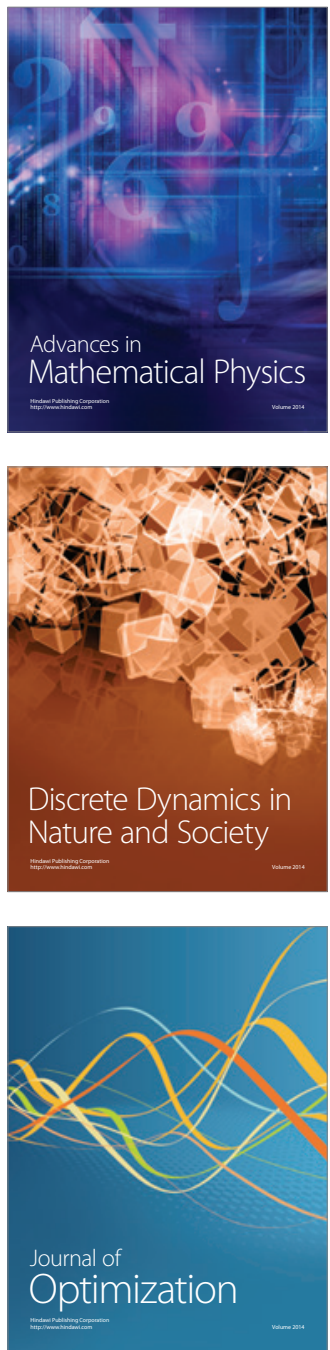